\title{
Análise da Vulnerabilidade do Tráfego Urbano usando Dados de Aplicativo de Ridesharing
}

\author{
Antônio Augusto de Faria G. M. Pertence ${ }^{1}$, Raquel A. F. Mini ${ }^{1}$ \\ Humberto Torres Marques-Neto ${ }^{1}$
}

${ }^{1}$ Programa de Pós-graduação em Informática Pontifícia Universidade Católica de Minas Gerais, Rua Walter Ianni, 255 - Belo Horizonte - Minas Gerais - CEP: 3198011 - Brasil

antonio.pertencedsga.pucminas.br, raquelminiepucminas.br

humberto@pucminas.br

\begin{abstract}
Complex network theory has been used to model the urban traffic of road systems. The urban traffic can be modeled as a graph characterized by complex network metrics. According to the literature, if there is a problem of traffic congestion or disruption, an evaluation of the degree of vulnerability or resilience of the system in the search for solutions is made. An approach for evaluation the vulnerability of urban traffic is proposed through the complex network metrics generated from an open data set obtained by the Ridesharing application Uber, using targeted failures. As a differential, in relation to the literature, this approach uses the skewness to assess the vulnerability of urban traffic.
\end{abstract}

Resumo. A teoria de redes complexas tem sido usada para modelar o tráfego de sistemas viários urbanos. O tráfego pode ser modelado como um grafo, caracterizado por métricas de redes complexas. Segundo a literatura, se há um problema de congestionamento ou interrupção do tráfego, é feita uma avaliação do grau de vulnerabilidade ou resiliência do sistema na busca de soluções. O artigo propõe uma abordagem para avaliação da vulnerabilidade do tráfego urbano através das métricas das redes complexas geradas a partir de dados abertos obtidas pelo aplicativo de Ridesharing Uber, utilizando falhas direcionadas. Como diferencial, em relação a literatura, esta abordagem utiliza o conceito de assimetria para a avaliação da vulnerabilidade do tráfego urbano.

\section{Introdução}

O sistema viário urbano tem grande importância para o funcionamento das cidades. É através dele que se faz a integração entre as diversas áreas econômicas e sociais, possibilitando a mobilidade da população que sai cotidianamente de áreas residenciais onde mora em busca das diversas áreas comerciais, industriais, de saúde e de educação. Assim, busca-se estabelecer condições de transporte que levem a um desempenho satisfatório do tráfego urbano e assegurem o pleno funcionamento da infraestrutura das cidades [Jenelius et al. 2015]. Para melhorar o tráfego urbano, os planejadores podem utilizar ferramentas matemáticas, tais como, o uso de otimização do sistema viário [Sebastiani et al. 2016] ou modelos probabilísticos para descrever o comportamento dos usuários [Vonu et al. 2011].

A teoria de redes complexas também tem sido usada para modelar o sistema de viário, uma vez que tem a vantagem de modelar interações complexas entre objetos em alta dimensionalidade. O tráfego do sistema viário urbano pode ser modelado como um 
grafo, caracterizado estatisticamente por meio de métricas de redes complexas. Através dos dados do aplicativo de Ridesharing são obtidas as regiões da cidade definidas como os nós e as conexões entre regiões adjacentes, como arestas, cujos pesos podem corresponder ao tempo de viagem entre as regiões conectadas [Pearson et al. 2017].

As métricas de redes complexas podem ser usadas para avaliar o grau de vulnerabilidade ou resiliência do sistema viário urbano através de observação de suas alterações frente a ocorrência de problemas de congestionamento e interrupção do tráfego em várias condições [Chen et al. 2011] e [Balijepalli et al. 2014]. Neste trabalho considera-se que a vulnerabilidade de uma rede complexa é a medida do decaimento do desempenho quando alguns os nós e ou suas respectivas conexões (arestas) são removidos [Latora e Marchiori 2001].

O objetivo do presente trabalho é a proposição de uma abordagem para a avaliação da vulnerabilidade do tráfego urbano, através das métricas das redes complexas geradas a partir de um conjunto de dados abertos das cidades americanas de Cincinnati, Orlando e Pittsburgh obtidas no aplicativo de Ridesharing Uber Movement [Uber 2019], durante o terceiro trimestre de 2019. A partir do cálculo de métricas locais da rede de cada cidade, foi feito o levantamento de métricas globais, considerando um processo progressivo de remoção dos nós a partir dos maiores valores de cada métrica local até a remoção completa. Também foi feito uma avaliação da assimetria dos resultados para melhor caracterizar o processo de inclusão de falhas direcionadas para a avaliação da vulnerabilidade na rede complexa. A escolha das métricas locais e globais foi baseada no trabalho de Ferber et al. (2012), e o conceito da assimetria dos resultados foi obtido em Nist (2003).

A abordagem proposta pode ser aplicada em outras cidades, independentemente do tamanho e localização das mesmas. Com ela, se consegue observar a vulnerabilidade, de uma forma geral, quando são introduzidas falhas direcionadas, pois, com o uso do conceito de assimetria aplicado neste trabalho, é possível avaliar se a falha acontece logo para os valores maiores das métricas ou não, ou seja, se há maior ou menor vulnerabilidade da rede. Em geral, na literatura, a análise de vulnerabilidade se baseia na somatória dos valores das métricas globais obtidas pela remoção de nós e arestas [Ferber et al. 2012]. A Seção 2 apresenta o referencial teórico, a Seção 3 os trabalhos relacionados, e as Seções 4, 5 e 6 apresentam respectivamente a abordagem proposta, resultados e conclusão.

\section{Referencial Teórico}

Esta seção apresenta os referenciais teóricos a partir dos quais a pesquisa foi desenvolvida, tais como: redes complexas suas métricas e vulnerabilidades, e os dados abertos do aplicativo de Ridesharing.

\subsection{Redes Complexas}

Uma rede complexa é um conjunto de nós conectados por um conjunto de arestas e pode modelar interações complexas entre objetos em alta dimensionalidade. Os nós destas redes representam as unidades fundamentais do sistema em questão e as arestas estabelecem quais dos nós estão conectados a outros.

Dentre os sistemas que assumem a forma de redes incluem-se a Internet, as redes sociais, as redes financeiras, as redes biológicas, as redes de infraestrutura, as redes de 
comunicação e muitas outras [Easley et al. 2010]. No sistema de Ridesharing são obtidas as regiões da cidade definidas como os nós e as conexões entre regiões adjacentes, como arestas [Pearson et al. 2017].

Se há um problema de congestionamento ou interrupção do tráfego, pode ser feita uma avaliação do grau de vulnerabilidade ou resiliência do sistema na busca de soluções. Segundo Latora e Marchiori (2001), a vulnerabilidade mede o decaimento do desempenho de uma rede complexa quando os nós e suas respectivas conexões (arestas) são removidos. Pode se trabalhar também com a ideia da resiliência ou robustez de uma rede complexa, em que se verifica a capacidade da rede em manter seu desempenho mesmo após perdas dos nós e arestas, também denominados de ataques.

Estes ataques podem variar desde falhas aleatórias, quando um nó ou uma conexão em uma rede é removido aleatoriamente, até um processo de destruição direcionada, em que os nós mais importantes de rede complexa são removidos. Nas redes complexas sem escala há uma grande quantidade de nós que possui baixo grau de conectividade. A remoção aleatória de nós pode ser feita em grande escala mantendo a rede conectada. Por outro lado, se for feito um ataque, considerando a eliminação dos nós mais importantes, ou seja, aqueles nós com maior grau, tal processo de eliminação direcionada destes poucos nós produzirá uma fragmentação da rede [Balijepalli 2014].

As características das redes complexas podem ser identificadas a partir de métricas que examinam as propriedades da vizinhança imediata dos nós, a medida do seu tamanho, sua interconectividade e as correlações dentro dessa vizinhança. Elas podem ser divididas em dois grupos: as métricas locais em que cada nó da rede possui valor próprio que pode ser diferente dos outros nós e as métricas globais, cujos valores, caracterizam a rede como um todo.

Em uma rede com peso, as arestas que conectam os nós são ponderadas na proporção de seu valor. A métrica de centralidade betweenness em uma rede com pesos é dada pela soma dos pesos de suas arestas adjacentes e é relacionada a medida de força dos nós indicada na Equação 1, sendo que $B_{i}$ é a centralidade betweenness do nó $i, N$ é o número de nós, $a$ é a matriz de adjacência e $w$ a matriz de peso entre os nós $i$ e $j$ respectivamente.

$$
B_{i}=\sum_{j=1}^{N} a_{i j} w_{i j}
$$

A métrica da centralidade betweenness foi escolhida para a avaliação da vulnerabilidade, pois quanto maior o seu valor, melhor será a conectividade entre os nós, gerando menor vulnerabilidade da rede [Barrat et al. 2004].

A métrica closeness de um nó é uma medida de centralidade em uma rede, calculada como recíproca da soma do comprimento dos caminhos mais curtos entre o nó e todos os outros nós no grafo como indica a Equação 2, sendo que $C_{i}$ é a centralidade closeness do nó $i, N$ é o número de nós, $d$ é a distância entre os nós $i$ e $j$ respectivamente.

$$
C_{i}=\frac{N}{\sum_{j=1}^{N} d_{i j}}
$$


A métrica da centralidade closeness também foi escolhida para a avaliação da vulnerabilidade, pois quanto maior seu valor, maior será a proximidade entre os nós, gerando menor vulnerabilidade da rede [Borgatti 2005].

Nas redes complexas, um coeficiente de agrupamento ou clustering é uma medida do grau em que os nós em um grafo tendem a se agrupar. O coeficiente de clustering local de um nó em um grafo, quantifica quão perto seus vizinhos estão de fornecerem um clique. Para grafos com pesos, existem várias maneiras de definir clustering. A definição usada neste trabalho é a média geométrica dos pesos das arestas dos subgrafos como indica a Equação 3, sendo que $A_{i}$ é o coeficiente de clustering do nó $i, N$ é o número de nós, $a$ é a matriz de adjacência e $w$ a matriz de peso entre os nós $i$ e $j$ respectivamente.

$$
A_{i}=\sqrt[N]{\prod_{j=1}^{N} a_{i j} w_{i j}}
$$

A métrica de clustering foi escolhida para a avaliação da vulnerabilidade, pois quanto maior o seu valor, maior a conectividade entre os nós, aumentando a resiliência, ou seja, diminuindo a vulnerabilidade da rede [Saramäki et al. 2007].

O conceito de eficiência local foi introduzido por Latora e Marchiori (2001) com o intuito de investigar redes reais. Supondo que uma rede complexa processe informações de forma paralela, isto é, cada nó pode enviar informações simultaneamente, ao longo da rede, a medida de eficiência local entre os nós $i$ e $j$ pode ser definida como o inverso da distância do caminho mais curto entre estes nós. A eficiência local de um dado nó $i$ é a média das eficiências entre os pares dos nós vizinhos deste nó $i$. Além disso, a eficiência local de uma rede é a média das eficiências locais dos nós desta rede. Para o presente trabalho, foram calculadas as eficiências locais dos nós da rede considerando somente os pares de nós que pertencem ao mesmo componente conexo, como indica a Equação 4, sendo que $E$ é a eficiência local, $N$ é o número de nós, $d$ é a distância entre os nós $i$ e $j$ respectivamente.

$$
E=\frac{1}{N(N-1)} \sum_{i=1}^{N} \sum_{j=1}^{N} \frac{1}{d_{i j}}
$$

Um componente conectado pode ser definido com um conjunto de nós em que há um caminho entre cada um deles. Em um grafo, o maior componente conectado é aquele com maior quantidade de nós. O tamanho relativo do maior componente conectado é a razão do seu tamanho e o número de nós do grafo. Para o contexto do transporte público, essa métrica foi escolhida para a avaliação da vulnerabilidade da rede, pois, pode indicar a abrangência de movimentação que um passageiro pode conseguir, comparando com uma rede sem arestas removidas [Ferber et al. 2012].

A assimetria é usada para avaliação da distribuição estatística dos resultados, como indicado na Equação 5, sendo que $S$ é a assimetria, $X_{i}$ é o valor do resultado $i, N$ número de resultados, $\mu$ média dos resultados e $\sigma$ desvio padrão dos resultados [Nist 2003].

$$
S=\frac{\sqrt{N(N-1)}}{N-2} \frac{\sum_{i=1}^{N}\left(X_{i}-\mu\right)^{3} / N}{\sigma^{3}}
$$


A avaliação da assimetria dos resultados indica que quando o seu valor é negativo, significa que a remoção dos nós com maior valor das métricas (betweenness, closeness e clustering), proporciona uma menor queda da métrica do grafo (eficiência, tamanho relativo maior componente conectado), indicando menor vulnerabilidade, tanto menor for o valor da assimetria e vice-versa [Joanes et al. 1998].

\subsection{Dados do Aplicativo de Ridesharing}

Uber Movement é uma ferramenta que compartilha informações sobre trânsito e mobilidade de algumas cidades onde o sistema Uber opera. A base de dados do Uber Movement é aberta ao público e pode ser baixado no formato CSV (arquivo de texto separado por vírgula), sem que sejam identificados o comportamento ou a identidade de usuários ou motoristas. $\mathrm{O}$ objetivo do Uber Movement é contribuir com o trabalho de gestores públicos, planejadores urbanos, pesquisadores e interessados em mobilidade. Usando informações obtidas com os dados, é possível avaliar o impacto da vulnerabilidade do tráfego urbano e fomentar diferentes políticas e tomadas de decisão sobre investimentos em infraestrutura [Uber 2019].

O Uber Movement calcula a duração média das viagens entre duas "zonas" em uma região por um determinado horário e data nas cidades disponíveis. As zonas definidas para uma região são comumente setores censitários, zonas de análise de tráfego ou bairros. Durante a viagem, registram-se periodicamente os rastros de GPS, que incluem latitude, longitude, data e hora. Esses rastros são comumente usados para fornecer roteamento de navegação, cálculos de tarifas, e combinar viagens entre motoristas e usuários. Quando agregados, esses rastros de GPS também podem ser usados para calcular os tempos médios de viagem entre as zonas em uma determinada região. A precisão dos dados é auxiliada pelo grande número de traços de GPS [Uber 2019].

\section{Trabalhos Relacionados}

No trabalho de Chen et al. (2012) é proposta uma abordagem de análise de vulnerabilidade através de uma "área de impacto" para avaliar as consequências do fechamento de estradas congestionadas dentro de uma certa área na cidade Hong Kong, ao invés de toda a rede. Os autores indicam que esta abordagem proposta pode reduzir significativamente o espaço de pesquisa para determinar os pontos mais críticos em redes de grande escala.

Diferentemente da abordagem desenvolvida no presente trabalho, que trabalha com uma base aberta de dados do Uber Movement, o trabalho de Chen et al. (2012) utiliza dados específicos da cidade de Hong Kong sobre estradas e não sobre regiões. Com a definição do índice de vulnerabilidade, os autores buscam avaliar o fluxo de tráfego nas regiões de impacto escolhidas, definindo os pontos mais críticos através de ataques direcionados (fechamento de estradas congestionadas).

O trabalho de Balijepalli et al. (2014) descreve vários índices de vulnerabilidade da literatura, e os aplica ao caso da rede urbana da cidade de York (Inglaterra), discutindo os resultados. Ele propõe um novo índice de vulnerabilidade considerando a avaliação da manutenção das conexões entre rodoviárias. Os autores usam os resultados do novo índice de vulnerabilidade para descrever um plano de desvio de tráfego, no caso de inundações na cidade de York, usando técnicas de modelagem de rede de tráfego combinadas com um sistema de informação geográfica. 
De forma diferente da abordagem desenvolvida no presente trabalho, o trabalho de Balijepalli et al. (2014) não trabalha com uma base aberta de dados de regiões do Uber Movement. Para a definição do índice de vulnerabilidade, eles utilizam a simulação de uma rede de fluxo de tráfego em estradas a partir de dados específicos de origem e destino, obtidos na cidade de York. Em seu trabalho, os autores buscam encontrar as vias mais problemáticos com relação a possibilidade de enchentes, avaliando o impacto da vulnerabilidade da rede na retirada dessas vias.

Utilizando o conjunto de dados do Uber Movement, Person et al. (2017) propõe um meio de modelar o fluxo de tráfego ao longo do dia, usando medidas de métricas de centralidade das redes complexas em uma sequência de grafos temporais direcionados com pesos dinâmicos. Ele analisa os gargalos de tráfego e os horários mais críticas do tráfego urbano em várias cidades, comparando esses grafos temporais em diferentes momentos do dia com um grafo espacial que modela as estruturas de cada cidade.

De forma semelhante, à abordagem desenvolvida no presente trabalho, Person et al. (2017) utiliza os dados abertos do Uber Movement [Uber 2019]. Porém, estes autores usam apenas métricas locais das redes complexas, enquanto no presente trabalho utilizam-se tanto as métricas locais, quanto as métricas globais. Eles buscam estudar quais são as regiões que tem o tráfego mais congestionado, trabalhando com dados temporais de hora em hora, não utilizando a técnica de ataques direcionados. No presente trabalho procura-se a avaliação da vulnerabilidade da rede de tráfego urbano como um todo utilizando a média consolidada de dados durante o dia, obtidas no Uber Movement, a partir de falhas direcionadas [Uber 2019].

\section{Abordagem Proposta}

Para o desenvolvimento da abordagem proposta para avaliação das vulnerabilidades usando dados de aplicativo de Ridesharing, através das métricas das redes complexas, foram escolhidas cidades que tinham a disponibilidade destes dados. Para as cidades escolhidas, os dados de tráfego urbano por rua do Uber Movement já estavam agregados por regiões [Uber 2019]. Foi criado um grafo simples para representar as regiões de cada cidade definidas como os nós e as conexões entre regiões adjacentes, como arestas. Os pesos destas arestas correspondem ao tempo de viagem entre as regiões conectadas [Pearson et al. 2017].

Foi feita a caracterização das redes complexas das cidades escolhidas, através do número de nós e arestas e após o cálculo das métricas betweenness, closeness, e clustering da rede para cada cidade, foi feito o levantamento de cada métrica global (eficiência e tamanho relativo do maior componente conectado) considerando um processo progressivo de remoção dos nós e suas respectivas arestas a partir dos maiores valores de cada métrica local até a remoção completa (a cada interação é retirada um porcentagem dos maiores valores), seguido da avaliação da assimetria dos resultados [Nist 2003].

Além do conjunto de dados abertos do Uber Movement, foram utilizadas as ferramentas computacionais como a biblioteca NetworkX escrita na linguagem Python utilizada para a criação, manipulação e estudo da estrutura, dinâmica e funções de redes complexas [Hagberg et al. 2008]. A Figura 1 ilustra os procedimentos utilizados para avaliação da vulnerabilidade por aplicativo Ridesharing. 


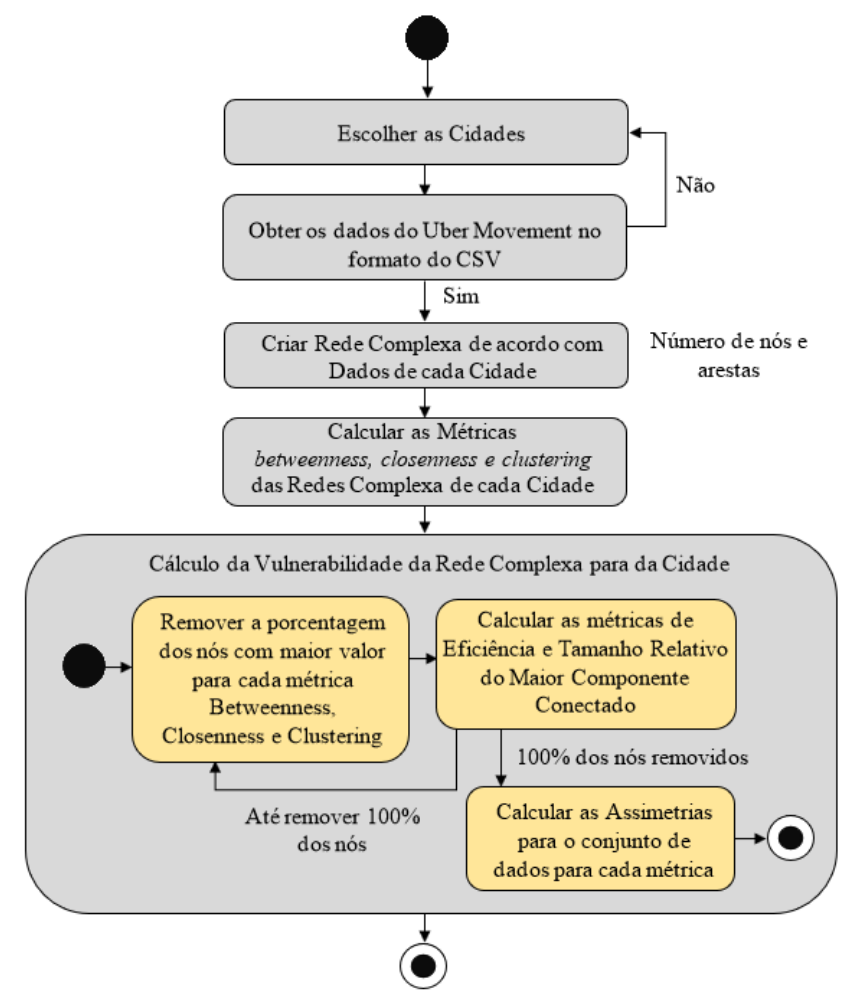

Figura 1. Procedimento de Avaliação da Vulnerabilidade da Rede para o Aplicativo Ridesharing.

\section{Resultados}

As cidades americanas de Cincinnati, Orlando e Pittsburgh foram escolhidas devido à disponibilidade de dados abertos do Uber Movement. As regiões de cada cidade foram definidas como os nós e as conexões entre regiões adjacentes, como arestas. As arestas da rede possuem como peso, o tempo de viagem entre as regiões conectadas. Para geração da rede de cada cidade escolhida, os arquivos do Uber Movement são lidos no formato $\mathrm{CSV}$, selecionando-se as viagens que foram feitas no terceiro trimestre de 2019. A Tabela 1 mostram o número de nós e arestas das redes complexas das três cidades.

Tabela 1. Número de Nós e Arestas das Redes Complexas

\begin{tabular}{|c|c|c|}
\hline Cidades & Número de Nós & Número de Arestas \\
\hline Cincinnati & 131 & 189 \\
\hline Orlando & 326 & 603 \\
\hline Pittsburgh & 587 & 1183 \\
\hline
\end{tabular}

Os dados da Tabela 1 mostram que a maior rede é a da cidade de Pittsburgh e a menor é da cidade de Cincinnati. Isto é devido ao fato de que a cidade de Cincinnati foi dividida em setores censitários maiores.

A Figura 2 indica as regiões obtidas a partir dos dados abertos do Uber Movement e seus respectivos grafos das redes de tráfego urbano para as cidades de Cincinnati, Orlando e Pittsburgh. 

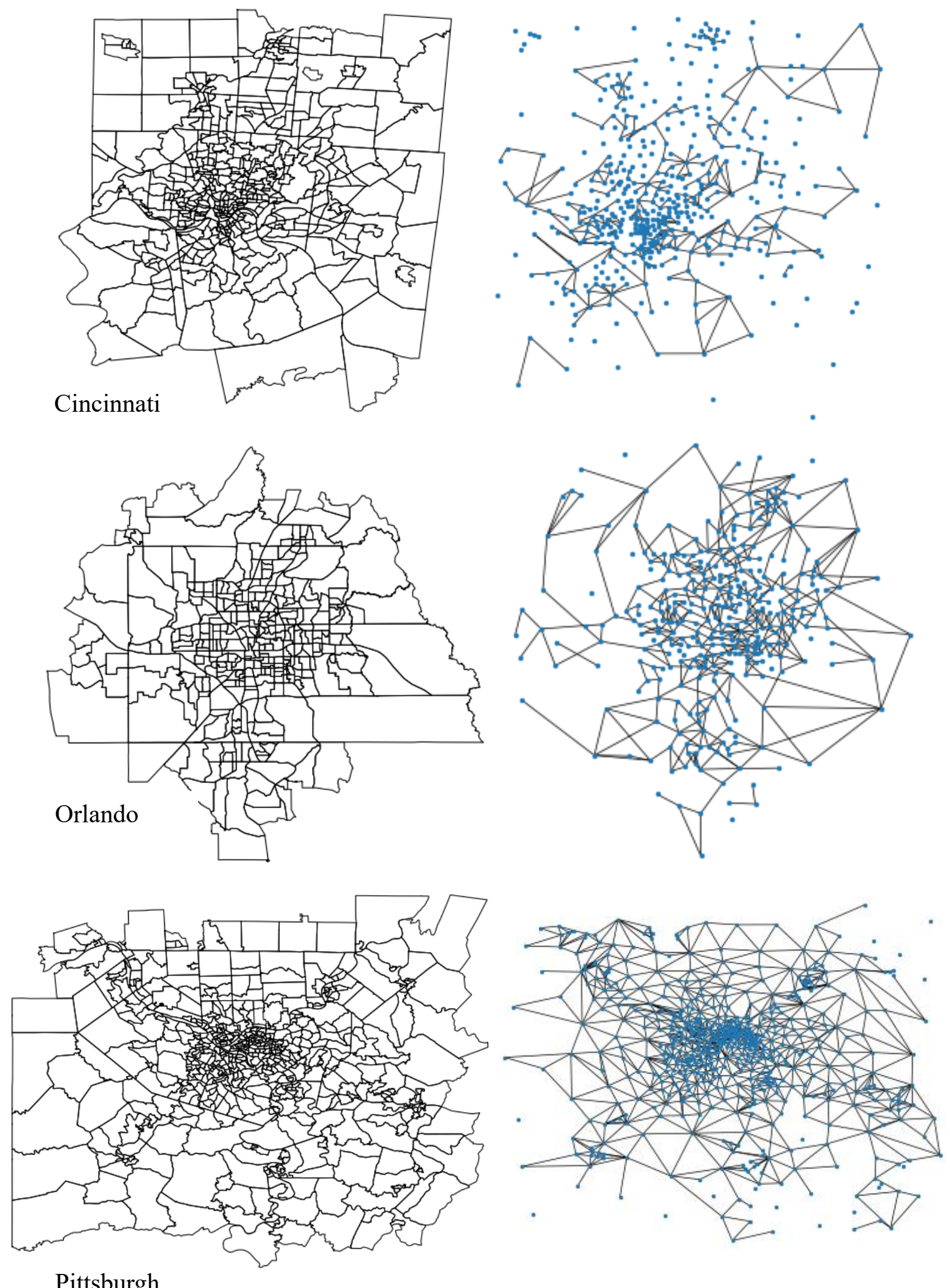

Figura 2. Regiões do Uber Movement e o Grafo da Rede de Tráfego Urbano para as cidades de Cininnati, Orlando e Pittsburgh. 
Observa-se nos grafos das redes de tráfego urbano das três cidades que nem todos os pontos que representam as regiões estão conectados por arestas, visto que não há informações disponíveis sobre estas regiões na base de dados abertos no Uber Movement.

A Figura 3 indica os histogramas da distribuição percentual dos nós, considerando a variação percentual dos valores das métricas locais de betweenness, closeness $e$ clustering para as cidades de Cincinnati, Orlando e Pittsburgh, sendo que $0 \%$ se refere ao menor valor e $100 \%$ ao maior valor obtido em cada métrica.
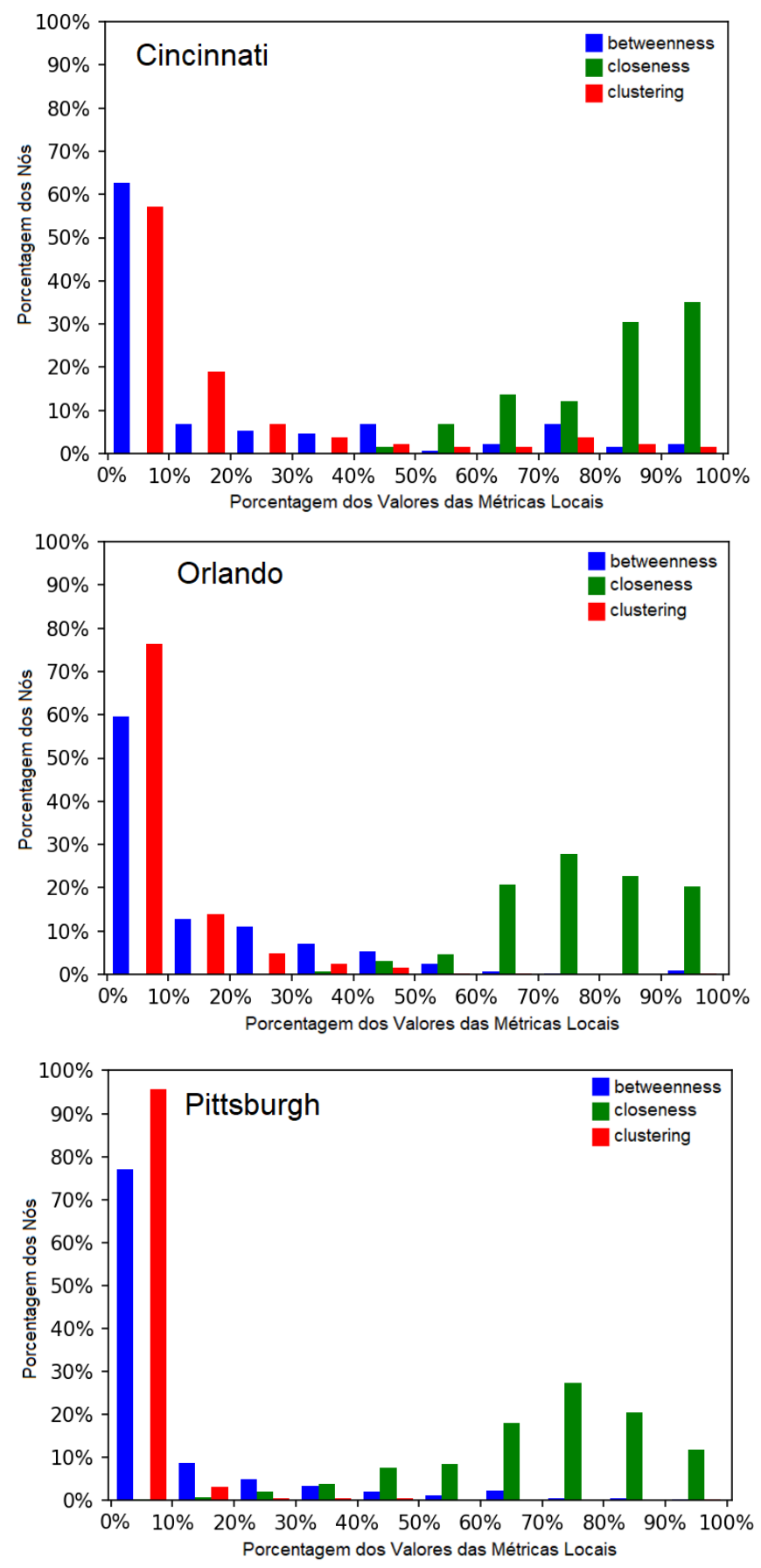

Figura 3. Histograma da Porcentagem dos Valores das Métricas Locais Betweenness, Closeness e Clustering para as cidades de Cincinnati, Orlando e Pittsburgh (Valores normalizados entre $0 \%$ e $100 \%$ ). 
Observa-se que no caso das métricas betweenness e clustering, a maioria dos nós se concentra nos percentuais de menor valor, enquanto que no caso da métrica de closeness, a porcentagem dos nós se distribuem entre os valores médios e maiores.

A Figura 4 indica os valores da métrica global de eficiência da rede, considerando as métricas locais betweenness, closeness e clustering, e suas respectivas assimetrias, em função da porcentagem de nós removidos para as cidades de Cincinnati, Orlando e Pittsburgh.
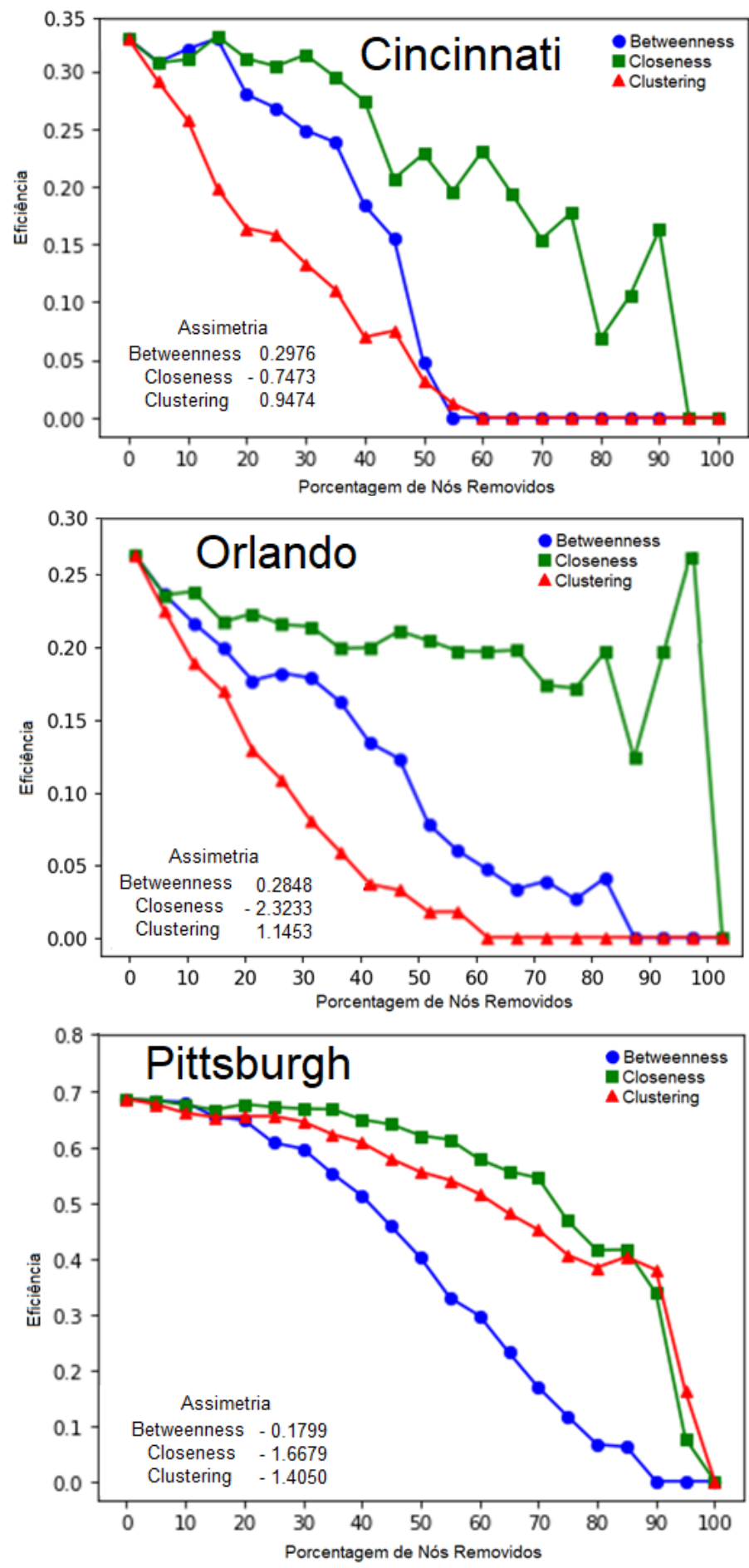

Figura 4. Eficiência x Porcentagem dos Nós Removidos para as cidades de Cincinnati, Orlando e Pittsburgh. 
A maior influência no decaimento para a métrica global da eficiência é dada pelas curvas da métrica de clustering para as cidades de Cincinnati e Orlando, e pela curva de betweenness para a cidade de Pittsburgh. Isto pode ser quantificado pelos valores de assimetria associados.

O fato de serem observados aumentos na eficiência da rede no caso das curvas de closeness para as cidades de Cincinnati e Orlando, não significa a redução da vulnerabilidade geral da rede destas duas cidades. Considerando ainda o caso de redes com vários componentes conexos pequenos, haverá maior eficiência, pois os caminhos serão bem curtos. Entretanto, em qualquer caso, com a remoção dos nós e suas arestas haverá uma fragmentação da rede, que estará menor e não mais atenderá as necessidades de tráfego urbano das cidades.

As Figuras 5a, 5b e 5c indicam os valores da métrica global do tamanho relativo do maior componente conectado da rede, considerando as métricas locais betweenness, closeness e clustering, e suas respectivas assimetrias, em função da porcentagem de nós removidos para as cidades de Cincinnati, Orlando e Pittsburgh.

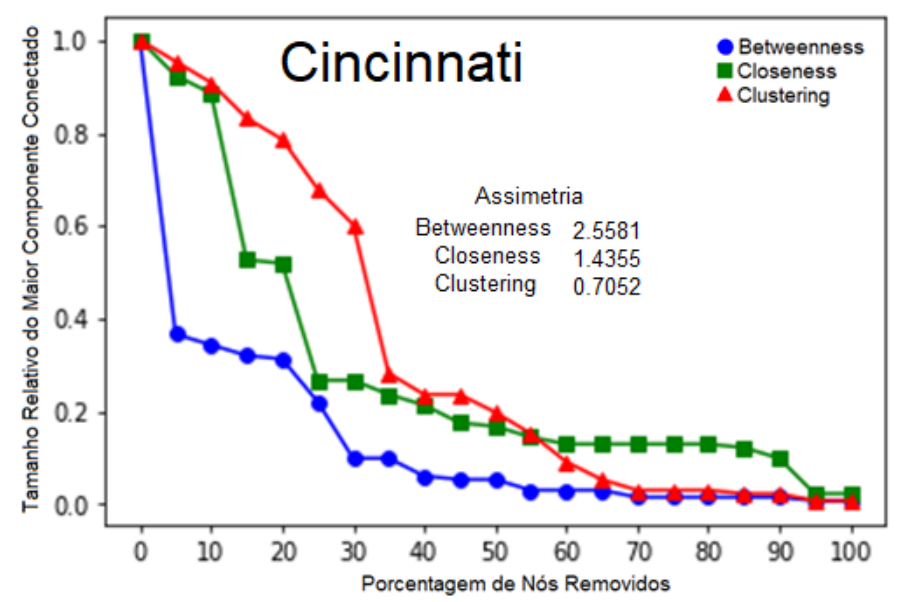

Figura 5a. Tamanho Relativo do Maior Componente Conectado x Porcentagem dos Nós Removidos para a cidade de Cincinnati

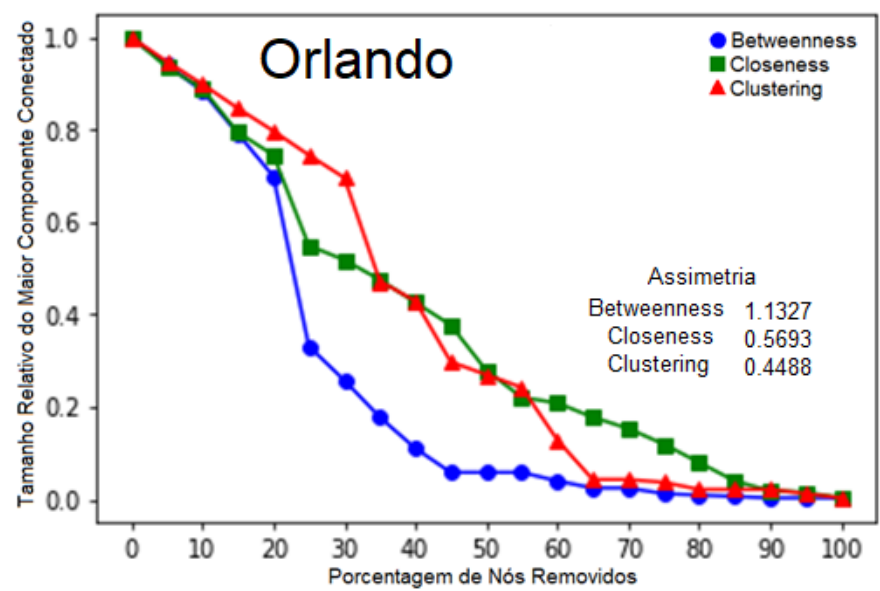

Figura 5b. Tamanho Relativo do Maior Componente Conectado x Porcentagem dos Nós Removidos para a cidade de Orlando 


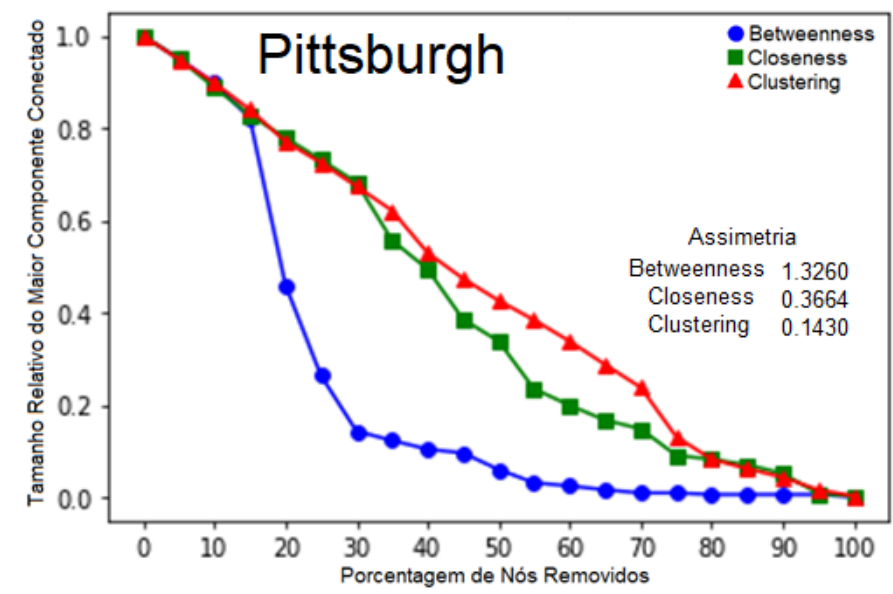

Figura 5c. Tamanho Relativo do Maior Componente Conectado x Porcentagem dos Nós
Removidos para a cidade de Pittsburgh

No caso da métrica global do tamanho relativo do maior componente conectado da rede, observa-se um comportamento semelhante para as três cidades. A métrica betweenness é a que mais influencia no decaimento no processo de remoção dos nós. A observação das curvas indica que o tamanho relativo do maior componente conectado apresenta maior decaimento a partir da remoção dos primeiros nós com os maiores valores desta métrica local. Isto também pode ser quantificado pelos valores de assimetria associados.

Barrat et al. (2004) e Saramäki et al. (2007) indicam que nos casos das métricas locais de betweenness e clustering, quanto menores os seus valores, pior será a conectividade entre os nós e maior vulnerabilidade da rede. Assim o fato das métricas locais de betweenness e clustering serem as métricas que mais influenciam na vulnerabilidade das métricas global da eficiência e do tamanho relativo do maior componente conectado da rede, vem de encontro ao fato de que a maioria dos valores dos nós para estas métricas locais se concentra nos percentuais de menor valor para as três cidades (Figura 3).

A retirada inicial do percentual dos nós e suas arestas com maiores valores produz um conjunto de nós não retirados que apresentam baixos valores, propiciando maior vulnerabilidade da rede, diferentemente da métrica local de closeness, cuja porcentagem dos nós se distribuem entre os valores médios e maiores. Neste caso, a retirada inicial do percentual dos nós e suas arestas com maiores valores não produz um conjunto de nós não retirados que apresentam baixos valores, mantendo a proximidade entre os nós e gerando menor vulnerabilidade da rede [Borgatti 2005].

Considerando a análise do tráfego urbano usando dados de aplicativo Ridesharing Uber Movement para as cidades de Cincinnati, Orlando e Pittsburgh, observa-se que as métricas globais estudadas se mostram adequadas para a avaliação da vulnerabilidade da rede, apresentando maior vulnerabilidade através da métrica de betweenness no caso do tamanho relativo do maior componente conectado para três cidades e para o caso da eficiência a maior vulnerabilidade relativa a métrica de clustering para as cidades de Cincinnati e Orlando e relativa a métrica de betweenness para a cidade de Pittsburgh, em consonância com Ferber et al. (2012). 
Entretanto, não é possível definir uma métrica global como a mais adequada para avaliar a vulnerabilidade, nem a princípio, qual das métricas locais mais influenciaria no decaimento de uma dada métrica global. A resposta para isso dependerá do estudo de cada caso. De qualquer forma, a utilização da abordagem proposta pode ser aplicada a outras cidades de uma maneira mais seletiva, investigando a vulnerabilidade da rede com métricas globais e locais que mais influenciam em cada caso.

\section{Conclusão}

A abordagem proposta pode ser aplicada em outras cidades, independentemente do tamanho e localização das mesmas, desde que se tenha acesso aos dados abertos dados do Uber Movement.

$\mathrm{Na}$ literatura a análise de vulnerabilidade se baseia na somatória dos valores das métricas globais obtidas pela remoção de nós e arestas [Ferber et al. 2012], ou ainda como no trabalho de Pearson et al. (2017) que utiliza apenas métricas locais das redes complexas, buscando estudar quais são as regiões que tem o tráfego mais congestionado, trabalhando com dados temporais de hora em hora, sem utilizar a técnica de ataques direcionados.

No presente trabalho procura-se a avaliação da vulnerabilidade da rede de tráfego urbano como um todo, utilizando a média consolidada de dados durante o dia, obtidas no Uber Movement a partir de falhas direcionadas. É possível diferenciar as condições de falha a partir a avaliação da assimetria de cada métrica global utilizada, identificando como as métricas locais influenciam na vulnerabilidade destas métricas globais, estabelecendo assim um melhor entendimento e diferencial nas condições dos casos estudados.

Em continuidade ao trabalho realizado, deseja-se aplicar a abordagem proposta às redes de transporte por ônibus, de forma separada e em conjunto com o sistema de aplicativo por Ridesharing visando buscar um melhor entendimento do sistema de transporte urbano como um todo [Uber 2019]. Além disso, a abordagem proposta poderá ser integrada a uma interface gráfica para ser utilizados por planejadores de transporte.

\section{Agradecimentos}

Este trabalho foi financiado pela FAPEMIG (PPM-00253-18), CNPq, CAPES e STIC AmSud 18-STIC-07.

\section{Referências}

Barrat, A., Barthélemy M., Pastor-Satorras R., Vespignani, A. (2004) The architecture of Complex Weighted Networks, Proceedings of the National Academy of Sciences, March 16, vol. 101, n. 11, pp 3747-3752

Balijepalli, Chandra, Oppong, Olivia (2014) Measuring vulnerability of road network considering the extent of serviceability of critical road links in urban areas, Journal of Transport Geography, 39, pp. 145-155. 
Borgatti, Stephen P. (2005) Centrality and network flow, Social networks ,27, pp. 55- 71.

Chen, Bi Yu, Lam Willian H. K., Sumalee, A., Li, Qingquan, Li, Zhi-Chun (2012) Vulnerability analysis for large-scale and congested road networks with demand uncertainty, Transportation Research Part A: Policy and Practice 46.3, pp. 501-516.

Easley, D., Jon K. (2010) "Networks, Crowds, and Markets", Vol. 8, Cambridge: Cambridge University Press.

Ferber, C., Berche, B., Holovatch, T., Holovatch Yu. (2012) A tale of Two Cities Vulnerabilities of the London and Paris Transit Networks, J Transp. Secur. 5. 199/216.

Hagberg, A., Swart, P., Chult, D. (2008) "Exploring Network Structure, Dynamics, and Function Using NetworkX". in Proceedings of the 7th Python in Science Conference (SciPy2008), pp. 11-15.

Jenelius, E., Mattsson L-G. (2015) Vulnerability and resilience of transport systems - A discussion of recent research, Transportation Research Part A, 81, pp. 16-34.

Joanes, D. N., Gill, C. A. (1998). Comparing measures of sample skewness and kurtosis Journal of the Royal Statistical Society: Series D (The Statistician) 47, pp. 183-189.

Latora, V., Marchiori, M. (2001) Efficient behavior of small-world networks. Physical review letters 87.19, pp.198701.

Nist (2003), "Engineering Statistics Hanbook", https://www.itl.nist.gov/div898/ handbook/eda/section3/eda35b.htm

Pearson, M., Sagastuy, J., Samaniego, S. (2017) "Traffic Flow Analysis Using Uber Movement Data", htts:// snap.stanford.edu/class/projects/

Saramäki, J., Kivel, M., Onnela, J-P., Kaski1, K., Kertész, J. (2007) Generalizations of the Clustering Coefficient to Weighted Complex Networks, Physical Review E, 75.2, pp. 027105.

Sebastiani, M., Luders R., Fonseca K. (2016) "Evaluating electric bus operation for a real-world BRT public transportation using simulation optimization," IEEE Trans. Intel. Transp. Sys., vol. 99, pp. 1-10.

Uber (2019) “Uber Movement”, https://movement.uber.com/

Vonu P., Tang L., Vassilakis W. (2011) "Spatio-temporal effects of bus arrival time information," in Proceedings of the 4th ACM SIGSPATIAL International Workshop on Computational Transportation Science, CTS '11, (New York, NY, USA), pp. 6-11. 